\title{
Survey of non-resuscitation fluids administered during septic shock: a multicenter prospective observational study
}

Anja Lindén-Sønders $\varnothing^{1}$, Mårten Jungner ${ }^{2}$, Martin Spångfors ${ }^{3}$, Mohammed Jan ${ }^{4,5}$, Adam Oscarson ${ }^{6}$, Sally Choi ${ }^{7}$, Thomas Kander ${ }^{5}$, Johan Undén ${ }^{6}$, Donald Griesdale ${ }^{8}$, John Boyd ${ }^{9}$ and Peter Bentzer ${ }^{1,10^{*}}$ (D)

\begin{abstract}
Background: The indication, composition and timing of administration of non-resuscitation fluid in septic shock have so far received little attention and accordingly the potential to reduce this source of fluid is unknown. The objective of the study was to quantify and characterize non-resuscitation fluid administered to patients with septic shock.

Methods: This prospective observational study was performed in eight intensive care units in Sweden and Canada during 4 months in 2018. Adult patients with septic shock within $24 \mathrm{~h}$ of admission to the intensive care unit were eligible for inclusion. Non-resuscitation fluids were defined as fluids other than colloids, blood products and crystalloids at a rate $\geq 5 \mathrm{ml} / \mathrm{kg} / \mathrm{h}$. Indication, volume and type of fluid were recorded during the first 5 days after admission. A maximum of 30 patients could be included per centre. To estimate the potential to reduce administration of nonresuscitation fluid, a pragmatic "restrictive" protocol for administration of non-resuscitation fluids was devised based on the most restrictive practice already in place for non-resuscitation fluids at any of the participating centres. Data are presented as median (interquartile range [IQR]).
\end{abstract}

Results: A total of 200 patients were included in the study and the 30-day mortality was $35 \%$. Patients received a total of 7870 (4060-12,340) ml of non-resuscitation fluids and 2820 (1430-4580) of resuscitation fluids during the observation period. Median volumes of non-resuscitation and resuscitation fluids were similar at day 1 (1620 [7102320] and 1590 [520-3000]) $\mathrm{ml}$, respectively) and non-resuscitation fluids represented the largest source of fluid from day 2 and onwards after admission to the ICU. Vehicles for drugs such as vasoactive drugs and antibiotics constituted the largest fraction of non-resuscitation fluids (2400 [1270-4030] $\mathrm{ml}$ ) during the 5-day observation period. Modelling suggested that volume of non-resuscitation fluids could be reduced by 2840 (1270-4900) $\mathrm{ml}$ during the first 5 days of admission to the ICU, mainly through reducing maintenance fluids.

Conclusions: Non-resuscitation fluids constitute the major fraction of fluids administered in the ICU to patients suffering from septic shock and may represent the largest modifiable target to reduce fluid overload.

Keywords: Septic shock, Non-resuscitation fluids, Fluid balance, Vehicle

\section{Background}

Administration of resuscitation fluids intravenously to ensure adequate tissue perfusion is a cornerstone in the treatment of sepsis and septic shock [1]. However, it is

\footnotetext{
*Correspondence: peter.bentzer@med.lu.se

1 Department of Anesthesiology and Intensive Care, Helsingborg Hospital, Charlotte Yhlens gata 10, 25187 Helsingborg, Sweden Full list of author information is available at the end of the article
}

increasingly recognized that excessive fluid administration has potentially detrimental side effects such as tissue edema with impaired oxygen delivery and compartment syndromes. Observational studies have suggested that a positive fluid balance is associated with poor outcome [2-4]. These observations have inspired studies which investigated whether conservative resuscitation strategies in patients with septic shock can improve survival or 
reduce the need for life support and associated complications [5-7].

There are many indications for fluid administration in the critically ill. Observational studies have shown that the majority of fluid administered to patients admitted to an intensive care unit (ICU) is for indications other than maintaining intravascular volume, such as nutrition or as a vehicle for intravenous medications $[4,8,9]$. This is perhaps not a surprising finding in hemodynamically stable patients, but several studies indicate that even in patients with septic shock, a major part of the fluid is given for indications other than volume expansion $[6$, 10-12]. Interestingly, non-resuscitation fluid appears to be the major source of fluid administered for septic shock already within the first few days of admission to the ICU, meaning that these fluids will contribute to the positive fluid balance often observed in the early phases of septic shock $[2,6]$.

The indication, composition and timing of administration of non-resuscitation fluid in septic shock have so far received little attention and accordingly the potential to reduce this source of fluid is unknown. Based on these considerations, the objective of the present study was to quantify and characterize the fluid given to patients with septic shock during the first 5 days of admission to the ICU and to assess the potential to reduce the non-resuscitation fluid, by modelling a restrictive fluid protocol. For this purpose, we conducted a prospective observational study in eight intensive care units in Sweden and Canada.

\section{Methods}

\section{Study design and ethics}

We conducted this multicenter observational study on eight different sites: six intensive care units in southern Sweden (Helsingborg, Halmstad, Lund, Kristianstad, Malmö and Varberg), and two in Vancouver, British Columbia, Canada (St Paul's Hospital and Vancouver General Hospital). Patients were included between March 1st 2018 and June 30th 2018. Follow-up time was 30 days from inclusion. The regional ethical boards in Lund, Sweden (application \# 2017/565) and Vancouver, Canada (\#H17-03504) approved the study. The ethical board in Sweden required informed consent from patients or their legal surrogates before enrollment, whereas the ethical board in Vancouver waived the need for informed consent. We prepared the manuscript according to the STROBE guidelines for observational studies and registered the study at Clinicaltrials.gov NCT03438097 prior to inclusion of the first patient.

\section{Patients}

We included consecutive adult patients ( $\geq 18$ years) with septic shock per SEPSIS-3 criteria [13] within $24 \mathrm{~h}$ of admission to respective ICU. Patients were excluded if they were previously included in the study on a prior admission.

\section{Data collection}

During the first 5 days of admission to the ICU, we registered fluid input, output and fluid balance. Nurses registered fluid input, output and fluid balance manually, in all sites but one. At this site, computer software collected fluid input and fluid balance electronically, while a nurse registered fluid output manually. We collected data on patient characteristics from the electronic medical records/paper records and the electronic records differed across the study sites. A designated researcher at each site transferred the data to the CRF. This researcher was carefully informed on how to register the data onto the CRF and was not blinded to the study objectives.

We specified fluids as either non-resuscitation fluids or resuscitation fluids. The non-resuscitation fluids were subdivided into six groups: vehicle for drugs, enteral and parenteral nutrition, glucose solutions, enteral water and crystalloid administered at a rate of $<5 \mathrm{ml} / \mathrm{kg} / \mathrm{h}$ [14]. The group 'vehicle for drugs' was further subdivided into type of drugs: vasoactive drugs (including inotropes), antibiotics, sedation, analgesics, insulin, potassium, other electrolytes and 'other drugs'. We defined resuscitation fluids as crystalloids administered at a rate of $\geq 5 \mathrm{ml} /$ $\mathrm{kg} / \mathrm{h}$, blood products or colloids and registered glucose solutions primarily given for nutritional purposes, i.e., concentrations of $10-20 \%$. Length of day 1 was calculated from time of admission to the ICU until change of day as defined at the respective site. In cases where ICU admission was less than 5 days, we defined the last day as the time from beginning of the last day until ICU discharge.

Daily fluid balance was determined by subtracting total fluid output, except for perspiration from total fluid intake. Bowel movements were included in the fluid balance according to local protocol. Demographic data were collected from the patient charts. Simplified Acute Physiology Score (SAPS-3) and Sequential Organ Failure Assessment (SOFA) were calculated on the day of admission. Mechanical ventilation or renal replacement therapy (RRT) during any of the first 5 days was registered, as well as source of sepsis and surgery as a source of infection. Length of stay in the ICU, ICU mortality and 30-day mortality were also recorded.

\section{Modelling of a restrictive fluid protocol}

In an attempt to estimate the potential to reduce administration of non-resuscitation fluid, we devised a pragmatic 
"restrictive" protocol for administration of non-resuscitation fluids based on the most restrictive practice already in place for non-resuscitation fluids at any of the participating centres. In this protocol, we assumed the following: no maintenance fluid was given to patients with a positive cumulative fluid balance, no intravenous glucose was given for nutritional purposes, and enteral nutrition was changed to a concentration of $2 \mathrm{kcal} / \mathrm{ml}$ in centres using less concentrated formulas.

\section{Statistics}

No sample size calculation was performed. Based on historical admission rates in the smaller participating ICUs during a typical 4-month period, a maximum inclusion of 30 patients per ICU was set in an attempt to balance the cohort. We performed statistical analyses using GraphPad Prism 8.1.1 (GraphPad Software, La Jolla, CA, USA) and R v. 3.5.2 (R Core Team, Vienna, Austria). We did not use imputation for missing data. Data are presented as medians and interquartile ranges.

\section{Results}

\section{Demographics}

We screened a total of 1946 consecutive patients for eligibility during the study period. Of these, 208 patients were diagnosed with septic shock within $24 \mathrm{~h}$ of admission. A total of 8 patients were not included (missing fluid charts $=3$, no consent $n=3$, death before consent could be obtained $n=2$ ) leaving 200 patients for inclusion in the analysis. Each centre included a median of 24 patients (min-max 16-30). The 30-day mortality in the cohort was 71/200 (35\%) and the most common source of sepsis was abdominal infection. Length of ICU stay was 78 (47-169) h. A detailed description of characteristics is presented in Table 1. Fluid data were missing from one patient on day 2 and 3 due to shift of care to palliation, from one patient on day 4 due to transfer to another ICU, and from one patient on day 5 for unknown reasons. Output data were incomplete in one patient due to lack of urinary catheter.

\section{Fluid administration}

Patients received a median of $7870(4060-12,340) \mathrm{ml}$ of non-resuscitation fluids and $2820(1430-4580) \mathrm{ml}$ of resuscitation fluids during the observation period. Daily intake of fluids and daily fluid balance are presented in Fig. 1. Median volumes of non-resuscitation- and resuscitation fluids on day 1 were similar, 1620 (710-2320) and $1590(530-3000) \mathrm{ml}(p=0.072$, Wilcoxon rank test), respectively. From day 2 and onwards, daily volume of non-resuscitation fluids was larger than daily volume
Table 1 Patient characteristics

\begin{tabular}{|c|c|c|}
\hline & & Missing data \\
\hline Number of patients & 200 & NA \\
\hline Female sex & $71(36)$ & 0 \\
\hline Age, years & $69(59-77)$ & 0 \\
\hline Weight at admission, kg & 78 (66-90) & 15 \\
\hline SAPS-3 & $73(64-82)$ & 30 \\
\hline SOFA score on day 1 & $10(8-12)$ & 0 \\
\hline $\begin{array}{l}\text { Highest lactate concentration on } \\
\text { day } 1, \mathrm{mmol} / \mathrm{l}\end{array}$ & $3.6(2.7-5.2)$ & 0 \\
\hline Length of ICU stay, h & $78(47-169)$ & 0 \\
\hline Alive at ICU discharge & $158(79.0)$ & 0 \\
\hline Alive at 30 days & $129(64.5)$ & 0 \\
\hline Renal replacement therapy & $48(24)$ & 0 \\
\hline Mechanical ventilation & $135(67.5)$ & 0 \\
\hline Surgery & $58(29)$ & 0 \\
\hline \multicolumn{3}{|l|}{ Source of sepsis } \\
\hline Abdominal & $71(35.0)$ & \\
\hline Respiratory & $70(34.5)$ & \\
\hline Soft tissue & $24(11.8)$ & \\
\hline Genitourinary & $19(9.4)$ & \\
\hline Cardiovascular & $3(1.5)$ & \\
\hline Central nervous system & $1(0.5)$ & \\
\hline Unknown & $15(7.4)$ & \\
\hline
\end{tabular}

Data are presented as median (IQR) or as number (\%). Mechanical ventilation and renal replacement therapy at any time during the study observation period. Surgery as a cause of sepsis or for source control

NA non-applicable

of resuscitation fluids. A daily breakdown of the different subgroups of non-resuscitation fluids is presented in Fig. 1a and Table 2. Vehicles for drugs constituted the largest fraction of non-resuscitation fluids during the observation period. These vehicles were mainly used for vasoactive drugs and antibiotics as shown in Fig. 1b. Daily fluid balance was positive during the first 3 days in the ICU and cumulative fluid balance was positive during the entire observation period, as demonstrated in Fig. 1c. Total volume of resuscitation and non-resuscitation fluid is presented per site in Fig. 2. There was significant variation in the use of each subgroup of non-resuscitation fluid between the different sites, except for parenteral nutrition (Additional file 1).

\section{The restrictive fluid protocol}

By modelling a restrictive protocol of non-resuscitation fluids in the respective centre, we obtained a theoretical reduction of 2840 (1270-4900) ml per patient of non-resuscitation fluids for the whole cohort, during the observation period (Additional file 2). We observed 

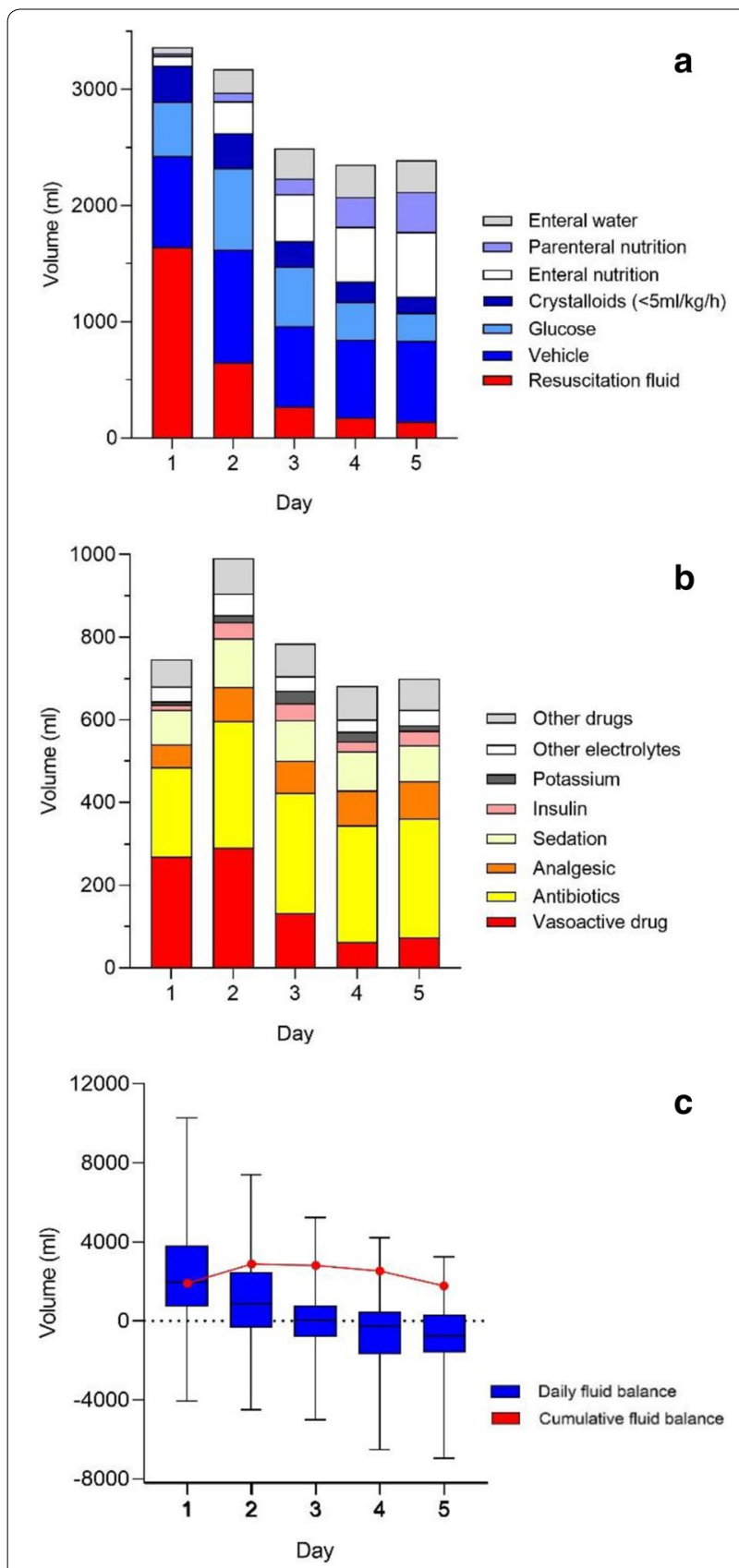

Fig. 1 a Median daily volume and type of fluids. Data for type of fluids each day are presented as fraction of total daily volume. Please note that sum of the median daily volume does not equal sums of median non-resuscitation and resuscitation fluids over the whole observation period because of the skewed distribution of the data. $N$ number of patients. $\mathbf{b}$ Median daily volume and type of vehicle. Data for type of vehicle each day are presented as fraction of total daily volume. N number of patients. c Daily and cumulative fluid balance. Daily fluid balance is presented as median, interquartiles and range, and cumulative fluid balance (dots) as median the largest reductions for glucose solutions in the Swedish centres and for crystalloids as maintenance fluids in nearly half of the Swedish and one of the Canadian centres (Additional file 3).

\section{Discussion}

In patients with septic shock, we demonstrated that the median volume of non-resuscitation and resuscitation fluids was similar at day 1 and that non-resuscitation fluids represented the largest source of fluid from day 2 and onwards after admission to the ICU. Daily fluid balance was positive until day 3 after admission. Fluid as a vehicle for intravenous drugs constituted the major contributor to non-resuscitation fluids.

Resuscitation fluids are administered rapidly to increase preload by an increase in intravascular volume, with crystalloids most commonly administered for this purpose. However, crystalloids may also be administered at a lower rate to provide hydration and to maintain homeostasis, commonly referred to as maintenance fluid. To separate these two indications, we defined a crystalloid as a non-resuscitation fluid if administered at a rate of less than $5 \mathrm{ml} / \mathrm{kg} / \mathrm{h}$. This definition aligns with several previous surveys on fluid administration practices $[8,12$, 14]. However, it should be noted that some of the studies mentioned below have used other definitions. For instance, crystalloids were defined as a non-resuscitation fluid if administered for reasons other than circulatory impairment [6] or as a resuscitation fluid regardless of infusion rate [7] and in some studies classification of crystalloids was not described in detail [4, 9]. Accordingly, fraction of crystalloids of the non-resuscitation fluids may differ somewhat and highlight the need for a consensus with regard to definitions. Because crystalloids are generally a small fraction of total volume of nonresuscitation fluid, this will not influence the conclusions below.

Previous studies surveying the use of non-resuscitation fluids in patients admitted to ICUs have included a broad set of critically ill patients regardless of their hemodynamic status and need of resuscitation fluids [4, $8,9]$. In this study, we focused our efforts on patients with septic shock, because this is a subgroup of critically ill patients in which the importance of fluid resuscitation is emphasized in guidelines and is also a group where positive fluid balance tends to be a clinical problem. Very little is known about use of non-resuscitation fluids in septic shock, but some data can be extracted from a pilot trial (CLASSIC), comparing two protocols 
Table 2 Daily volume of fluids

\begin{tabular}{lcllccccc}
\hline $\begin{array}{l}\text { Day } \\
\text { Resuscitation } \\
\text { fluid }\end{array}$ & Vehicle & $\begin{array}{l}\text { Parenteral } \\
\text { nutrition }\end{array}$ & $\begin{array}{l}\text { Enteral } \\
\text { nutrition }\end{array}$ & Enteral water & $\begin{array}{l}\text { Crystalloids }<5 \text { ml/ } \\
\mathbf{k g} / \mathbf{h}\end{array}$ & $\begin{array}{l}\text { Glucose } \\
\begin{array}{l}\text { Total non- } \\
\text { resuscitation } \\
\text { fluid }\end{array}\end{array}$ \\
\hline 1 & $1590(525-3000)$ & $640(290-1000)$ & $0(0-0)$ & $0(0-0)$ & $0(0-40)$ & $0(0-590)$ & $210(0-860)$ & $1620(710-2320)$ \\
2 & $400(0-1260)$ & $820(390-1240)$ & $0(0-0)$ & $0(0-350)$ & $0(0-190)$ & $0(0-580)$ & $590(0-1390)$ & $2580(1560-3450)$ \\
3 & $60(0-500)$ & $500(230-1010)$ & $0(0-0)$ & $130(0-620)$ & $110(0-300)$ & $0(0-410)$ & $210(0-890)$ & $2220(1290-2930)$ \\
4 & $0(0-270)$ & $490(200-1010)$ & $0(0-290)$ & $160(0-670)$ & $120(0-350)$ & $0(0-200)$ & $0(0-480)$ & $2240(1390-2930)$ \\
5 & $0(0-200)$ & $570(310-970)$ & $0(0-610)$ & $300(0-800)$ & $160(0-350)$ & $0(0-50)$ & $0(0-290)$ & $2160(1700-2840)$ \\
\hline
\end{tabular}

Volumes are presented as median (IQR). Please note that the sum of the daily medians of the different components of non-resuscitation fluids does not equal the median of the daily total volume of non-resuscitation fluids due to the skewed distribution of the data

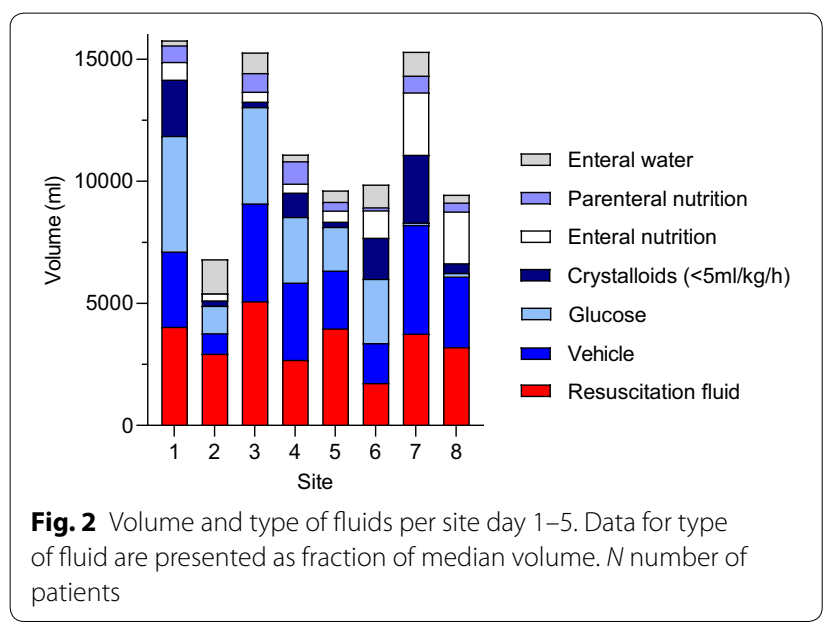

for administration of resuscitation fluids in septic shock [6]. In that study, patients were included approximately $4 \mathrm{~h}$ after admission to the ICU and non-resuscitation fluids constituted the major part of the administered fluid already on day 1 . Our finding show that this is true also if the early phase of ICU admission, during which resuscitation fluids are most likely to be administered, is included in the day 1 data. The daily fluid balance remained positive up to the 3rd day after admission in our study, in line with previous data from patients with septic shock [6]. With regard to total volume of nonresuscitation fluid during the 5-day observation period, the two studies differ substantially. Patients in the present cohort received approximately $8 \mathrm{l}$ and patients in CLASSIC cohort received approximately $11 \mathrm{l}$. Differences may be explained by variations in local practice but could also reflect a general change in practice patterns due to increasing awareness of potential adverse effects of intravenous fluids. Another possible explanation is that the median length of stay in the present study was 3 days compared to 6 days in the CLASSIC cohort. Other recent multicenter studies of cohorts of mixed ICU patients reported the total volume of non-resuscitation fluids during the first 3 days of admission to be 5-5.5 1 which is similar to our finding of $6.0 \mathrm{l}$ during the same time interval [4, 9]. Similar to our results, these studies also reported that vehicles were the largest fraction of the non-resuscitation fluids.

Based on the growing concern for the adverse effects of excessive fluid administration, several recent pilot studies have assessed if administration of resuscitation fluids can be reduced by "restrictive" protocols or by prediction of fluid responsiveness prior to administration of fluids $[5-7,15]$. Data from these studies suggest that the volume of resuscitation fluid can be reduced by 0.8 to $1.2 \mathrm{~L}$ during the first $3-5$ days of ICU admission. Our results, suggesting that administration of nonresuscitation fluid theoretically could be reduced by a median of $2.8 \mathrm{l}$ during the first 5 days in the ICU, indicate that this approach could potentially have an even larger impact on fluid balance in the ICU.

Several aspects of our modelling could be considered. First, the choice to omit the use of maintenance fluids or glucose could be questioned. However, we are not aware of any studies supporting the use of maintenance fluids in patients that are in a positive fluid balance, nor are we aware of any studies or guidelines suggesting that intravenous glucose should be administered during the acute phase of critical illness [16, 17]. Second, our data suggested that a large part of the vehicles were administered as diluents of antibiotics and vasoactive drugs. To model the potential to reduce vehicles for antibiotics, one could consider both the potential to reduce vehicle volume in already used antibiotics as well as a shift away from antibiotics requiring large volumes of vehicle. Because of the large number of different antibiotics and very limited data on solubility and safety of concentrated antibiotic solutions, such modelling would have been very complex and we therefore elected not to do it. Similarly, we did not model potential reductions in administration of vasoactive drugs, because this group of drugs consists of several different 
individual drugs with different potentials for concentration. This means that we could have underestimated the potential to reduce the volume of non-resuscitation fluids. Third, the assumption that reductions in volume of non-resuscitation fluid would not be offset by increased administration of resuscitation fluids could be questioned, since crystalloid maintenance fluids will also distribute in the intravascular space. However, intravascular retention of crystalloids over time is most likely very low and is reported to be $<10 \%$ in inflammatory conditions meaning that this source of error is reasonably small $[18,19]$. Lastly, the physicians caring for the included patients may have aimed for a positive cumulative fluid balance when prescribing non-resuscitation fluids in patients with a perceived preexisting fluid deficit. If so, we may have overestimated the potential to reduce the volume of non-resuscitation fluids somewhat in those patients.

The considerable variation between sites regarding the administration of the different subtypes of non-resuscitation fluids observed in the present study aligns with recently reported data from a multicenter retrospective study in centres in the UK and Canada [4]. This indicates that local practice traditions, rather than evidencebased medicine, play a major role in determining volume as well as the type of non-resuscitation fluid, and highlight the need for more knowledge in this aspect of fluid therapy. Interestingly, none of the sites had any written guidelines with regard to intravenous administration of maintenance fluids or glucose solutions in septic shock. The high variability of current practice has its implications on the design of interventional trials, due to the difficulties in defining a common baseline to which an intervention can be compared. Nevertheless, we believe that our results provide a rationale for an interventional study in which a more restrictive approach of administration of non-resuscitation fluids can be compared to current practice.

While ongoing trials are addressing if restricting resuscitation fluids in septic shock impacts survival [20,21], an important aspect to bear in mind is that the balance between benefit and harm when reducing resuscitation fluids may be different than the balance when reducing non-resuscitation fluids. Thus, it is important that any intervention with the objective to reduce administration of non-resuscitation fluids should be rigorously assessed in trials regardless of the findings in the ongoing resuscitation fluid trials.

\section{Strengths and limitations}

Strengths of our study include that data are contemporary and collected from multiple sites in both university and regional hospitals. Moreover, the study was also prospectively designed and data were consecutively gathered with high granularity.

Limitations include that even though every effort was made to ensure that fluid input data were captured in a similar way at the different sites, we cannot exclude that smaller amounts of fluids were not registered or that subtle differences in collection of data may have contributed to the inter-site variability seen in the results. Also, we did not standardize registration of bowels movements in the fluid balance which may have resulted in an overestimation of fluid balance in some centres. Other limitations include the small sample size and that only two countries participated in the study. This may limit the validity of our findings in other countries.

\section{Conclusions}

Non-resuscitation fluids constitute the major fraction of fluids administered in the ICU to patients suffering from septic shock and may represent the largest modifiable target to reduce fluid overload.

\section{Supplementary information}

Supplementary information accompanies this paper at https://doi. org/10.1186/s13613-019-0607-7.

Additional file 1. Sources of fluid input day $1-5$ by study site. Volumes are presented in millilitres, length of stay presented in hours (median [IQR]). *Kruskal-Wallis test.

Additional file 2. Volume and type of fluid during day $1-5$ in "standard care" vs "restrictive" protocol. Volumes are presented in millilitres (median $[\mathrm{IQR}])$

Additional file 3. Potential non-resuscitation fluid reduction during day $1-5$, per site, in the "restrictive" protocol. Volumes are presented in millilitres (median [IQR]). Please note that the sum of the medians does not equal median of the sum because of the skewed distribution of data.

Abbreviations

ICU: Intensive care unit; IQR: Interquartile range; SAPS III: Simplified Acute Physiology Score, 3rd edition; SOFA: Sequential organ failure assessment; RRT: Renal replacement therapy.

\section{Authors' contributions}

AL: data collection, statistical analysis, study coordination, and manuscript drafting. MJu: study design, data collection and manuscript drafting. MS, JU, TK, DG and JB: study conduct, data collection and manuscript revision. MS, AO, SC and MJa: data collection manuscript revision. PB: principal investigator, study design, study coordination and manuscript drafting. All authors read and approved the final manuscript.

Funding

PB is funded by grants from Region Skåne, Sweden (\# 18401), the Anna and Edwin Berger Foundation, Sweden and the Birgit and Henry Knutsson Foundation, Sweden. Open access funding provided by Lund University.

Availability of data and materials

The data is available from the corresponding author on reasonable request.

Ethical approval and consent to participate

All procedures were in accordance with the ethical standards of the institutional research committees and with the 1964 Helsinki declaration and its 
later amendments. The regional ethical boards in Lund, Sweden (application \#2017/565) and Vancouver, Canada (\#H17-03504) approved the study. Informed consent from patients or their legal surrogates before enrollment was required in Sweden whereas the ethics board in Vancouver, Canada waived the need for informed consent.

\section{Consent for publication}

Not applicable.

\section{Competing interests}

The authors declare that they have no competing interests.

\section{Author details}

${ }^{1}$ Department of Anesthesiology and Intensive Care, Helsingborg Hospital, Charlotte Yhlens gata 10, 25187 Helsingborg, Sweden. ${ }^{2}$ Department of Intensive and Perioperative Care, Skåne University Hospital, Malmö, Sweden. ${ }^{3}$ Department of Clinical Sciences Lund, Anesthesiology and Intensive Care, Kristianstad Hospital, Lund University, Lund, Sweden. ${ }^{4}$ Anesthesia Department, College of Medicine, Taibah University, Madinah, Saudi Arabia. ${ }^{5}$ Department of Anesthesia and Intensive Care, Skåne University Hospital, Lund, Sweden. ${ }^{6}$ Department of Operation and Intensive Care, Hallands Hospital, Halmstad, Sweden. ${ }^{7}$ Faculty of Medicine, University of British Columbia, Vancouver, BC, Canada. ${ }^{8}$ Department of Anesthesiology, Pharmacology \& Therapeutics, University of British Columbia, Vancouver, BC, Canada. ${ }^{9}$ Centre for Heart Lung Innovation, University of British Columbia, Vancouver, BC, Canada. ${ }^{10}$ Department of Clinical Sciences Lund, Anesthesiology and Intensive Care, Helsingborg Hospital, Lund University, Lund, Sweden.

Received: 3 July 2019 Accepted: 17 November 2019 Published online: 27 November 2019

\section{References}

1. Rhodes A, Evans LE, Alhazzani W, Levy MM, Antonelli M, Ferrer R, et al. Surviving sepsis campaign: international guidelines for management of sepsis and septic shock: 2016. Intensive Care Med. 2017;43:304-77.

2. Boyd JH, Forbes J, Nakada TA, Walley KR, Russell JA. Fluid resuscitation in septic shock: a positive fluid balance and elevated central venous pressure are associated with increased mortality. Crit Care Med. 2011;39:259-65.

3. Payen D, de Pont AC, Sakr Y, Spies C, Reinhart K, Vincent JL, et al. A positive fluid balance is associated with a worse outcome in patients with acute renal failure. Crit Care. 2008;12:R74.

4. Silversides JA, Fitzgerald E, Manickavasagam US, Lapinsky SE, Nisenbaum $R$, Hemmings $N$, et al. Deresuscitation of patients with iatrogenic fluid overload is associated with reduced mortality in critical illness. Crit Care Med. 2018;46:1600-7.

5. Richard JC, Bayle F, Bourdin G, Leray V, Debord S, Delannoy B, et al. Preload dependence indices to titrate volume expansion during septic shock: a randomized controlled trial. Crit Care. 2015;19:5.

6. Hjortrup PB, Haase N, Bundgaard H, Thomsen SL, Winding R, Pettilä V, et al. Restricting volumes of resuscitation fluid in adults with septic shock after initial management: the CLASSIC randomised, parallel-group, multicentre feasibility trial. Intensive Care Med. 2016;42:1695-705.

7. Corl K, Prodromou M, Merchant R, Gareen I, Marks S, Banerjee D, et al. The restrictive IV Fluid Trial in Severe Sepsis and Septic Shock (RIFTS): a randomized pilot study. Crit Care Med. 2019;12:951-9.
8. Bihari S, Teubner DJ, Prakash S, Beatty T, Morphett M, Bellomo R, et al. Fluid bolus therapy in emergency department patients: indications and physiological changes. Emerg Med Australas. 2016;28:531-7.

9. Van Regenmortel N, Verbrugghe W, Roelant E, Van den Wyngaert T, Jorens PG. Maintenance fluid therapy and fluid creep impose more significant fluid, sodium, and chloride burdens than resuscitation fluids in critically ill patients: a retrospective study in a tertiary mixed ICU population. Intensive Care Med. 2018;44:409-17.

10. Perner A, Haase N, Guttormsen AB, Tenhunen J, Klemenzson G, Åneman A, et al. Hydroxyethyl starch 130/0.42 versus Ringer's acetate in severe sepsis. N Engl J Med. 2012;367:124-34.

11. Bihari S, Prakash S, Bersten AD. Post resuscitation fluid boluses in severe sepsis or septic shock: prevalence and efficacy (price study). Shock. 2013;40:28-34.

12. Finfer S, Liu B, Taylor C, Bellomo R, Billot L, Cook D, et al. Resuscitation fluid use in critically ill adults: an international cross-sectional study in 391 intensive care units. Crit Care 2010;14:R185.

13. Singer $M$, Deutschman CS, Seymour CW, Shankar-Hari M, Annane D, Bauer M, et al. The Third International Consensus Definitions for Sepsis and Septic Shock (Sepsis-3). JAMA. 2016;315:801-10.

14. Hammond NE, Taylor C, Finfer S, Machado FR, An Y, Billot L, et al. Patterns of intravenous fluid resuscitation use in adult intensive care patients between 2007 and 2014: an international cross-sectional study. PLoS ONE. 2017;12:e0176292.

15. Cronhjort M, Bergman M, Joelsson-Alm E, Divander MB, Jerkegren E, Balintescu A, Mårtensson J, Svensen C. Fluid responsiveness assessment using passive leg raising test to reduce fluid administration and weight gain in patients with septic shock. J Anesth Perioper Med. 2017;4:169-78.

16. Singer P, Blaser AR, Berger MM, Alhazzani W, Calder PC, Casaer MP, et al. ESPEN guideline on clinical nutrition in the intensive care unit. Clin Nutrit. 2018;38:48-79.

17. McClave SA, Taylor BE, Martindale RG, Warren MM, Johnson DR, Braunschweig $C$, et al. Guidelines for the provision and assessment of nutrition support therapy in the adult critically ill patient: society of critical care medicine (SCCM) and American society for parenteral and enteral nutrition (A.S.P.E.N.). Crit Care Med. 2016;44:390-438.

18. Ernest D, Belzberg AS, Dodek PM. Distribution of normal saline and $5 \%$ albumin infusions in cardiac surgical patients. Crit Care Med. 2001;29:2299-302.

19. Statkevicius S, Frigyesi A, Bentzer P. Effect of ringers acetate in different doses on plasma volume in rat models of hypovolemia. Intensive Care Med Exp. 2017;5:50

20. Meyhoff TS, Hjortrup PB, Møller MH, Wetterslev J, Lange T, Kjaer MN, et al. Conservative vs liberal fluid therapy in septic shock (CLASSIC) trial-Protocol and statistical analysis plan. Acta Anaesthesiol Scand. 2019;63:1262-71.

21. SelfWH, Semler MW, Bellomo R, Brown SM, deBoisblanc BP, Exline MC, et al. Liberal versus restrictive intravenous fluid therapy for early septic shock: rationale for a randomized trial. Ann Emerg Med. 2018;72:457-66.

\section{Publisher's Note}

Springer Nature remains neutral with regard to jurisdictional claims in published maps and institutional affiliations. 